\title{
A coupling algorithm for partitioned solvers applied to bubble and droplet dynamics
}

\author{
Joris Degroote $^{\mathrm{a}}$, Peter Bruggeman ${ }^{\mathrm{b}}$, Jan Vierendeels ${ }^{*, \mathrm{a}}$ \\ ${ }^{a}$ Ghent University, Department of Flow, Heat and Combustion Mechanics, \\ St.-Pietersnieuwstraat 41, B-9000 Ghent, Belgium \\ ${ }^{\mathrm{b}}$ Ghent University, Department of Applied Physics, Jozef Plateaustraat 44, B-9000 \\ Ghent, Belgium
}

\begin{abstract}
Bubbles and droplets both consist of a liquid in contact with a gas. In this paper, we consider the interface between the incompressible liquid and the gas as a zero thickness structure. The position of the interface is determined by the equilibrium between surface tension effects and the fluid pressure difference across the interface. So, the structure interacts with the fluids on either side. The behaviour of a limited number of bubbles and droplets can therefore be simulated as a Fluid-Structure Interaction (FSI) problem.

Most existing techniques frequently used for studying bubble and droplet dynamics, such as Level Set or Volume Of Fluid, use monolithic schemes. The flow on both sides of the interface and the position of the interface are calculated in a single code. In this contribution, a partitioned approach is presented. The position of the interface is calculated with a structural solver. Given a displacement of the interface, a separate flow solver calculates the flow on the liquid side of the interface with the Arbitrary Lagrangian Eulerian (ALE) technique. The structural solver
\end{abstract}


uses a reduced order model of the flow solver to obtain implicit coupling between both solvers. This reduced order model is built up during the coupling iterations of a time step. Grid and time converged solutions of two axisymmetric problems are calculated: an oscillating water droplet in air and the growth and detachment of an air bubble from the outlet of a vertical needle, submerged in quiescent water.

Key words: Bubble, Droplet, Sharp interface, Fluid-Structure Interaction, Modal analysis, Reduced order model

PACS: 65M12, 65Z05, 76D45, 76T10

\section{Introduction}

Bubbles and droplets both consist of a liquid in contact with a gas. In this paper, we consider the interface between the incompressible liquid and the gas as a zero thickness structure. The interface position is calculated by a structural solver given the pressure on the liquid side of the interface. The latter is calculated by a flow solver given the position of the interface. This is very similar to Fluid-Structure Interaction (FSI) calculations. Therefore a short overview of methods frequently used to calculate bubble and droplet dynamics is given, followed by an overview of basic FSI coupling algorithms, before the new coupling algorithm is presented.

* Corresponding author.

Email address: Jan.Vierendeels@UGent. be (Jan Vierendeels).

$U R L$ : www.floheacom. ugent. be (Jan Vierendeels). 


\subsection{Bubbles and droplets}

Numerous techniques exist to simulate bubbles and droplets, and more in general multiphase flow. We limit ourselves to the techniques for a limited number of bubbles and droplets.

Front Capturing techniques do not track the interface with grid nodes. All phases are treated simultaneously and the conservative form of the NavierStokes equations is solved, so discontinuous physical properties at the interface between the phases are allowed. A difficulty for these methods is to maintain a sharp interface. Each of the following Front Capturing techniques differently determines with which fluid a cell is filled. The Marker And Cell (MAC) method uses marker particles to indicate fluid in a cell [13]. The Volume Of Fluid (VOF) method uses a marker function to store the mass fraction of one fluid currently residing in that cell $[15,34,19]$. The Level Set method uses the zero level of a smooth function to indicate the interface [29]. All these markers are transported with the flow. The Constrained Interpolation Profile method also transports the spatial derivatives of the marker function to reduce diffusion of the interface [48]. Front Capturing techniques often use the Continuum Surface Force model [2] to convert the surface tension into a volume force or the Continuum Surface Stress model [20] to convert it into a stress tensor.

Front Tracking techniques use grid nodes to track the interface. Ryskin et al. [36] use separate boundary fitted grids for each phase. Glimm et al. [12] use a fixed Eulerian grid and a moving Lagrangian interface and solve the governing equations of each phase separately. Tryggvason et al. [42] also work with an 
Eulerian grid and a Lagrangian interface, but they solve the Navier-Stokes equations simultaneously for all phases and add the surface tension on the interface with the Dirac $\delta$-function. The Boundary Integral Method (BIM) can be used for either inviscid [17] or Stokes flow [33] and has the advantage that only on the interface an equation has to be solved. In the Vortex-In-Cell (VIC) method, the interface is a vortex sheet moving over an Eulerian grid $[41,49]$.

Lagrangian techniques use a grid that deforms with the flow. The Particle Finite Element Methode (PFEM) uses the Lagrangian framework [28]. An Arbitrary Lagrangian-Eulerian (ALE) approach combines the advantages of the Eulerian and Lagrangian approach: it permits complex fluid motion and still maintains a sharp interface. An overview of the ALE description is given by Donea et al. [6] and ALE calculations of droplets have been performed by Deng et al. [4].

A completely different group are the lattice methods. Particles move from node to node on an Eulerian grid with a very small spatial scale and with small time steps. The rules for particle collision are adapted to model the physics of the flow. This behaviour asymptotically simulates the incompressible NavierStokes equations according to Frisch et al. [10]. An overview of lattice methods for two-phase flow with surface tension is given by Rothman et al. [35].

For simulations with frequent changes of topology such as break up of bubbles or droplets, the Front Capturing techniques and lattice methods are more suitable than Front Tracking and Lagrangian techniques because the latter have a deforming grid that has to be adapted to changes of topology. However, by positioning grid points on the interface, the Front Tracking and Lagrangian 
techniques represent the interface more accurately with a relatively coarse grid. In this work, change of topology is absent or limited but an accurate representation of the interface is wanted so an ALE approach is used.

\subsection{Fluid-Structure Interaction}

Although some of these methods treat the phases separately or use a fixed grid with a Lagrangian interface, they normally use a monolithic solver. In this paper, the interface between the gas and liquid phase is represented as a zero thickness structure and use separate solvers for the liquid flow and this structure. The position $X$ of the interface due to the fluid load $P$ on the interface is calculated with a structural solver $X=S(P)$. A flow solver $P=F(X)$ is used to calculate the fluid behaviour given the position of the interface. The simulation of the bubble or droplet behaviour is here considered as a problem involving FSI with partitioned solvers. Note that the flow solver calculates the flow in the entire liquid domain and it returns only the fluid load on the interface. Only the variables common to the fluid and the structure are mentioned in $X$ and $P$ as only those variables are needed for the coupling; other variables are hidden.

The main advantages of a partitioned scheme are the use of existing state-ofthe-art solvers for each aspect of the calculation and the possibility to switch to other solvers easily. The partitioned approach to FSI is the opposite of the monolithic approach where fluid and structure are calculated within one code $[1,18]$. A recent partitioned approach to FSI with free surfaces is given by Wall et al. [46]. We now describe some basic partitioned coupling techniques $[5,9,11,14,44]$. 
The coupling between the solvers in a partitioned FSI scheme can be explicit, implicit or a combination, irrespective of the explicit or implicit solution within the solvers. Explicit coupling means that the flow solver uses $X^{n}$ to calculate $P^{n+1}$ and the structural solver uses $P^{n}$ to obtain $X^{n+1}$. The superscript $n$ indicates the time step. The equilibrium between fluid and structure is therefore not guaranteed by explicit coupling. Explicit coupling is only stable when the interaction between fluid and structure is weak and is therefore often used in aeroelastic applications [8]. If the interaction is strong, e.g. with an incompressible liquid, implicit coupling is needed. The flow solver uses $X^{n+1}$ as input to calculate $P^{n+1}$ and the structural solver uses $P^{n+1}$ to obtain $X^{n+1}$. As the time scale of the fluid problem is often smaller than the time scale of the structural problem, one time step of the structural problem can be divided in smaller steps for the fluid problem. This is called subcycling [30]. For simplicity, the same time step is used for fluid and structure in this work. The time step is chosen based on the requirements of the flow solver as the time step does not appear in the structural solver.

The design of the coupling scheme can be serial or parallel, with or without coupling iterations $[23,37]$. For one parallel call to the solvers (Figure 1a), one calls the flow solver and the structural solver with the data from the previous time step and therefore this is fully explicit. For one serial call to the solvers (Figure 1b), the displacement of the structure due to the fluid load of the previous time step is calculated first, and then the flow that results from the new displacement is determined. This is explicit coupling for the structure and implicit for the fluid.

When iterative coupling is used, it is verified whether the equilibrium between the fluid and the structure is reached in every time step. Fixed point iterations 
can be used in a parallel (block Jacobi) or serial (block Gauss-Seidel) way. The coupling scheme shown in Figure 1c uses multiple parallel calls to the solvers, the scheme in Figure 1d multiple serial calls. Both are implicit coupling schemes, however explicit stepping is used: the load on the interface is constant during the calculation of the interface position and vice versa. Aitken-like underrelaxation increases the performance [26] and artificial compressibility of the liquid can stabilize the coupling [45]. Newton iterations can perform better than fixed point iterations, but they require the exact or approximate Jacobians of the solvers $[23,24,25]$. However, the Jacobians of the solvers are unavailable if the solvers are not accessible. Several other schemes have been developed from these basic schemes $[31,32,50]$.

To avoid instability of the coupling between a fluid and a light and flexible structure, the mass of the structure can artificially be increased [39]. Causin et al. [3] show with a simplified model of an artery that an incompressible fluid works on the structure as an added mass. According to Causin et al., the importance of the added mass effect increases if the density of the structure decreases and the length of the domain increases. Gerbeau et al. [11] use a reduced model for the flow solver, which enables them to capture the added mass effect. The reduced model is a linear inviscid model for the fluid, so they solve a flow problem that approximates the real flow problem through the liquid domain. In aeroelastic applications, a reduced order model of the flow solver can be constructed before the FSI simulation. This reduced order model is then coupled with the structure model to perform the FSI simulation [47].

In this paper we use implicit coupling with implicit stepping of the pressure on the interface in the coupling iterations: the structural solver uses a reduced order model $\hat{P}=\hat{F}(X)$ of the flow solver during the calculation of the interface 
position to have an approximate Jacobian of $F$ and to update $P$. This reduced order model is built up during the coupling iterations, with the responses from the real flow solver. This coupling scheme is illustrated in Figure 2.

In Section 2, the structural solver and the flow solver are defined. Only axisymmetric problems without mass transfer between the liquid and the gas are considered. Section 3 gives an overview of the coupling algorithm. The application of the method to an oscillating water droplet in air is presented in Section 4.1. The formation of an air bubble at the outlet of a vertical needle, submerged in quiescent water in the gravitational field is presented in Section 4.2 .

\section{Solvers}

\subsection{Structural solver}

\subsubsection{Surface tension}

As only axisymmetric bubbles and droplets are considered in this work, the interface is represented by a curve in a meridional plane. This curve is discretised with $N$ nodes. The nodes of the structure coincide with the nodes on the boundary of the liquid domain. On an interface, the condition

$$
\left(p_{1}-p_{2}+\sigma \kappa\right) \vec{n}=\left(\mathcal{T}_{1}-\mathcal{T}_{2}\right) \cdot \vec{n}+\nabla_{S} \sigma
$$

must be satisfied. A subscript 1 is used to indicate the fluid inside the bubble or droplet, 2 is used for the surrounding fluid. $p$ is the pressure, $\sigma$ the surface tension coefficient, $\kappa$ the local surface curvature, $\vec{n}$ the unit normal to the 
surface pointing outwards, $\mathcal{T}$ the viscous stress tensor and $\nabla_{S}$ the surface gradient. For the examples considered in this paper, we neglect the viscous forces on the interface and Marangoni effects, so we use

$$
p_{1}-p_{2}+\sigma \kappa=0
$$

These approximations yield accurate results, as will be shown in Sections 4.1 and 4.2 .

The interface becomes a free slip boundary because the viscous forces on the interface are neglected. Consequently, the nodes which represent the interface can be moved arbitrarily along the interface and the velocity of the fluids tangential to the interface is totally unrelated to the velocity of the interface nodes tangential to the interface.

\subsubsection{Curvature calculation}

The surface curvature is defined with the principal radii of curvature $R_{1}$ and $R_{2}$, which are positive if the interface is convex from the point of view of the fluid inside the bubble or droplet.

$$
\kappa=\frac{1}{R_{1}}+\frac{1}{R_{2}}
$$

For the calculation of $R_{1}$ and $R_{2}$ in node $i(i=1, \ldots, N)$ of the interface, a local parametric description of the interface is constructed with polynomial interpolants.

$$
\begin{aligned}
& x(s)=c_{0}+c_{1} s+c_{2} s^{2}+c_{3} s^{3}+c_{4} s^{4} \\
& r(s)=d_{0}+d_{1} s+d_{2} s^{2}+d_{3} s^{3}+d_{4} s^{4}
\end{aligned}
$$


with $x$ the axial coordinate, $r$ the radial coordinate and $s$ the arc length along the curve that represents the interface, starting from $s=0$ at node $i-2$. An index $i$ outside the range $1, \ldots, N$ denotes a ghost node, which represents a node that is mirrored around the axis of symmetry. In every node $i$, an iterative procedure is used to calculate the arc length $s$ and a set of coefficients $c_{j}, d_{j}$ $(j=0, \ldots, 4) . s$ is initialized with the length of the line segments between the nodes. Every iteration starts with the calculation of the coefficients $c_{j}$ in Eq. (4a) by solving

$$
\left[\begin{array}{c}
x_{i-2} \\
x_{i-1} \\
x_{i} \\
x_{i+1} \\
x_{i+2}
\end{array}\right]=\left[\begin{array}{ccccc}
1 & s_{i-2} & s_{i-2}^{2} & s_{i-2}^{3} & s_{i-2}^{4} \\
1 & s_{i-1} & s_{i-1}^{2} & s_{i-1}^{3} & s_{i-1}^{4} \\
1 & s_{i} & s_{i}^{2} & s_{i}^{3} & s_{i}^{4} \\
1 & s_{i+1} & s_{i+1}^{2} & s_{i+1}^{3} & s_{i+1}^{4} \\
1 & s_{i+2} & s_{i+2}^{2} & s_{i+2}^{3} & s_{i+2}^{4}
\end{array}\right]\left[\begin{array}{l}
c_{0} \\
c_{1} \\
c_{2} \\
c_{3} \\
c_{4}
\end{array}\right]
$$

with $s_{i-2}$ the arc length in node $i-2$, used for the parametric description of the interface around node $i$. Analogously for the coefficients $d_{j}$. The next step is calculating the new arc length $\hat{s}$ by treating $s$ as an ordinary parameter. 


$$
\begin{aligned}
\hat{s}_{i-2} & =0 \\
\hat{s}_{i-1} & =\hat{s}_{i-2}+\int_{s_{i-2}}^{s_{i-1}} \sqrt{\left(\frac{\mathrm{d} x}{\mathrm{~d} s}\right)^{2}+\left(\frac{\mathrm{d} r}{\mathrm{~d} s}\right)^{2}} \mathrm{~d} s \\
\hat{s}_{i} & =\hat{s}_{i-1}+\int_{s_{i-1}}^{s_{i}} \sqrt{\left(\frac{\mathrm{d} x}{\mathrm{~d} s}\right)^{2}+\left(\frac{\mathrm{d} r}{\mathrm{~d} s}\right)^{2}} \mathrm{~d} s \\
\hat{s}_{i+1} & =\hat{s}_{i}+\int_{s_{i}}^{s_{i+1}} \sqrt{\left(\frac{\mathrm{d} x}{\mathrm{~d} s}\right)^{2}+\left(\frac{\mathrm{d} r}{\mathrm{~d} s}\right)^{2}} \mathrm{~d} s \\
\hat{s}_{i+2} & =\hat{s}_{i+1}+\int_{s_{i+1}}^{s_{i+2}} \sqrt{\left(\frac{\mathrm{d} x}{\mathrm{~d} s}\right)^{2}+\left(\frac{\mathrm{d} r}{\mathrm{~d} s}\right)^{2}} \mathrm{~d} s
\end{aligned}
$$

The integrals in Eqs. (6) are calculated with Boole's rule. The new arc length $\hat{s}$ is now used to calculate a new parametrisation with Eq. (5). Three iterations are sufficient to obtain good accuracy for the arc length in the cases presented in this paper.

The derivatives of the parametric description Eqs. (4) evaluated in node $i$ are used to calculate the principal radii of curvature.

$$
\begin{aligned}
& R_{1}=\frac{\left[\left(\frac{\mathrm{d} x}{\mathrm{~d} s}\right)^{2}+\left(\frac{\mathrm{d} r}{\mathrm{~d} s}\right)^{2}\right]^{\frac{3}{2}}}{\frac{\mathrm{d} x}{\mathrm{~d} s} \frac{\mathrm{d}^{2} r}{\mathrm{~d} s^{2}}-\frac{\mathrm{d} r}{\mathrm{~d} s} \frac{\mathrm{d}^{2} x}{\mathrm{~d} s^{2}}} \\
& R_{2}=-r \frac{\sqrt{\left(\frac{\mathrm{d} x}{\mathrm{~d} s}\right)^{2}+\left(\frac{\mathrm{d} r}{\mathrm{~d} s}\right)^{2}}}{\frac{\mathrm{d} x}{\mathrm{~d} s}}
\end{aligned}
$$

On the axis of symmetry, a numerical singularity arises during the calculation of $R_{2}$, as both $r$ and $\frac{\mathrm{d} x}{\mathrm{~d} s}$ become zero. Limits and L'Hôpital's rule show that $R_{2}$ converges to $R_{1}$ as the distance to the axis approaches zero. So, on the axis the surface curvature $\kappa$ is defined as

$$
\kappa=\frac{2}{R_{1}}
$$

To test the curvature calculation, the curvature of half an ellipsoid with semi- 
axes of $1.5 \mathrm{E}-3 \mathrm{~m}$ and $1.0 \mathrm{E}-3 \mathrm{~m}$ has been calculated both analytically and numerically. The maximal, minimal and root mean square value of the relative error over the surface are shown in Figure 3. The relative error is the absolute value of the difference between the numerical and the analytical curvature, divided by the inverse of the long semi-axis. If $N$ represents the number of nodes, the maximal error decreases as $N^{2}$, the root mean square error as $N^{3}$ and the minimal error as $N^{4}$.

\subsubsection{Solver definitions}

The left hand side of Eq. (2) in node $i(i=1, \ldots, N)$ is called $g_{i}$. The first condition that must be satisfied in every unconstrained node of the interface is given by

$$
g_{i}=0
$$

with $i=1, \ldots, N$, except for constrained nodes.

The position of the nodes along the interface is arbitrary as stated in Section 2.1.1. To minimize the distortion of the fluid mesh surrounding the interface, the nodes are kept equidistant by a second set of equations

$$
\left[\left(x_{i}-x_{i-1}\right)^{2}+\left(r_{i}-r_{i-1}\right)^{2}\right]-\left[\left(x_{i}-x_{i+1}\right)^{2}+\left(r_{i}-r_{i+1}\right)^{2}\right]=0,
$$

with $i=1, \ldots, N$, except for constrained nodes. It is possible to make the position of the nodes on the interface dependent on the curvature by adding weights related to the curvature to both terms in the previous equation. Although this would be beneficial for the calculation of the curvature, it could be detrimental for the flow solver as highly curved parts of the interface are not necessarily more important for the flow and therefore this is not used in 
this work. The left hand side of Eq. (10) is further called $h_{i}$ and with this notation, Eq. (10) becomes

$$
h_{i}=0
$$

The first and the last node $(i=1, N)$ are constrained in the examples presented in this paper, but also other nodes can be constrained. If a constrained node $i$ has one degree of freedom, $g_{i}$ is the standard expression and $h_{i}$ is defined as

$$
a \cdot x_{i}+b \cdot r_{i}+c
$$

with $a, b$ and $c$ constants. If a node $i$ is fixed, both $g_{i}$ and $h_{i}$ are replaced by an expression as Eq. (12).

The expressions $g_{i}$ and $h_{i}$ with $i=1, \ldots, N$ are placed together in an array $S$.

$$
S=\left[g_{1} h_{1} \ldots g_{i} h_{i} \ldots g_{N} h_{N} f\right]^{\mathrm{T}}
$$

The expression $f(t)=t-\left(t^{n}+\Delta t^{n+1}\right)$ stating that the time $t$ does not vary during the time step is added. This enables the use of data from previous time steps in the reduced order model, as is explained later. To satisfy Eqs. (9) and (11), $S$ must equal zero.

$$
S(X, P)=0
$$

with $X$ the array that contains the node coordinates and the time $t . P$ is the array with the node values of the pressure on the liquid side of the interface. 


$$
\begin{aligned}
& X=\left[\begin{array}{llllll}
x_{1} & r_{1} \ldots x_{i} & r_{i} & \ldots & x_{N} r_{N} t
\end{array}\right]^{\mathrm{T}} \\
& P=\left[\begin{array}{llll}
p_{1} \ldots & p_{i} \ldots & p_{N}
\end{array}\right]^{\mathrm{T}}
\end{aligned}
$$

The code that solves Eq. (14) for the position of the interface $X$ is called the structural solver.

\subsection{Flow solver}

The pressure distribution on the liquid side of the interface $P$ is required, but also the gas pressure has to be known.

In this work, the gas pressure is modelled uniform in space. The pressure gradient in the gas is due to inertia, gravity and viscous stresses. As the density and viscosity of a gas are often a hundred to a thousand times lower than those of a liquid, we neglect the pressure variation on the gas side of the interface with respect to the variation on the liquid side. However, in cases where this assumption is not valid, it would be no problem for the presented method to compute the gas dynamics as well.

The pressure of the gas surrounding a liquid droplet is modelled constant. The pressure inside a bubble is calculated from the mass, temperature and volume $(X)$ of the bubble with the ideal gas law. The gas pressure is calculated within the structural solver.

In order to demonstrate the general applicability of the method, a black box code (Fluent 6.1, Fluent Inc.) is used to determine the pressure on the liquid side of the interface. Any code capable of computing the pressure distribution 
on the liquid side of the interface, given a deformation of this interface, can be used. The interface is modelled as a free slip wall since tangential stresses are assumed to be negligible. Its position is not known a priori. The ALE formulation of the Navier-Stokes equations is used to calculate the incompressible liquid flow. The grid nodes in the liquid domain close to the interface move along with the interface. A smaller fraction of the interface's displacement is applied as the distance to the interface increases.

The code that calculates the pressure distribution on the liquid side of the interface $P$ for a given position of the interface $X$, is called the flow solver, indicated with $F$.

$$
P=F(X)
$$

\section{Coupling algorithm}

Successive calls of the structural solver to obtain a new position of the interface and the flow solver to obtain a new distribution of the pressure on the liquid side of the interface without underrelaxation, lead to divergence of the coupling iterations within the time step for the examples presented in Sec-

tion 4. Iterative coupling of the solvers with explicit stepping in the coupling iterations of a time step fails due to the strong interaction between the incompressible liquid and the structure. Therefore, the structural problem, Eq. (14), has to be solved with an implicit treatment of the pressure on the liquid side of the interface. We use Newton's method to solve Eq. (14) for $X$ with implicit calculation of $P$. This requires the Jacobian of $F$. As the flow solver used here is inaccessible, this Jacobian is unavailable. So, a reduced order model of the 
flow solver is constructed based on modal analysis. An approximation of the Jacobian can be obtained from this reduced order model and the pressure distribution on the liquid side of the interface can be updated during the Newton iterations in the structural solver.

The new coupling procedure is now explained in detail. The values of $X^{n+1}$ and $P^{n+1}$ are calculated, starting from the known values of $X^{n}, P^{n}, U^{n}$ and $U^{n-1}$ (if available), with the superscript $n+1(n=0, \ldots)$ indicating the current time level. $U$ contains the time derivative (indicated with an overdot) of all the elements in $X$.

$$
U=\left[\dot{x}_{1} \dot{r}_{1} \ldots \dot{x}_{i} \dot{r}_{i} \ldots \dot{x}_{N} \dot{r}_{N} 1\right]^{\mathrm{T}}
$$

A subscript $k+1(k=0, \ldots)$ is used to indicate the current coupling iteration.

- First coupling iteration $(\mathrm{k}=0)$

A first guess for the position of the interface at $t^{n+1}$ is determined with an explicit scheme, third order accurate if the time step is constant.

$$
X_{1}^{n+1}=X^{n}+\left(\frac{3}{2} U^{n}-\frac{1}{2} U^{n-1}\right) \Delta t^{n+1}
$$

with $\Delta t$ the time step. The initial velocity is zero, so in the first time step $(n=0)$ we use $X_{1}^{1}=X^{0}$ with the initial position $X^{0}$ and initial pressure distribution $P^{0}$ given. In the second time step $(n=1)$, we use $X_{1}^{2}=X^{1}+$ $U^{1} \Delta t^{2}$, with $\Delta t^{2}$ the duration of the second time step. For the computed examples, the number of coupling iterations decreased by less than $2 \%$ when we used the third order scheme instead of a second order scheme, so higher order does not make sense here.

The pressure distribution on the liquid side of the interface is obtained from the flow solver. 


$$
P_{1}^{n+1}=F^{n+1}\left(X_{1}^{n+1}\right)
$$

The superscript of the flow solver indicates that the boundary conditions are updated to the time level $n+1$.

\section{- Further coupling iterations $(k \geq 1)$}

At the start of coupling iteration $k+1, k$ positions of the interface are known at $t^{n+1}$, with the corresponding distributions of the pressure on the liquid side of the interface. But also data from the previous $l$ time steps can be reused, with $l \geq 0$. If the time step is sufficiently small, the data of the previous time steps are still relevant. They are reused to make a better reduced order model and as a result to reduce the number of coupling iterations. We include the time $t$ in the array $X$ to make a decomposition based on the position of the interface and the corresponding time.

The following pairs of the interface position and the corresponding load on the liquid side are known:

$$
\left(X_{i}^{n+1-j}, P_{i}^{n+1-j}\right)
$$

with $i=1, \ldots, k$ for $j=0$ and if $l>0$ then also with $i=1, \ldots, k_{\text {last }}^{n+1-j}$ for $j=1, \ldots, \min (l, n)$. The index $k_{\text {last }}^{n+1-j}$ indicates the last coupling iteration of time step $n+1-j$. We limit $j$ by the minimum of $l$ and $n$ to ensure that no values before the start of the simulation are required. We indicate the total number of pairs by

$$
m= \begin{cases}k & \text { if } l=0 \\ k+\sum_{j=1}^{\min (l, n)} k_{\text {last }}^{n+1-j} & \text { if } l>0\end{cases}
$$

and notate the pairs as

$$
(X, P)_{i}
$$


with $i=1, \ldots, m$. Pair $m$ contains the data of coupling iteration $k$ at $t^{n+1}$. We want to convert pairs $(X, P)_{i}, i=1, \ldots, m-1$ into pairs $\left(V_{i}, W_{i}\right)$ of displacement $V_{i}$ relative to $X_{k}^{n+1}$ and change in pressure distribution $W_{i}$ relative to $P_{k}^{n+1}$. We therefore subtract pair $m$ from pairs $1, \ldots, m-1$.

$$
\left(V_{i}, W_{i}\right)=(X, P)_{i}-(X, P)_{m}
$$

with $i=1, \ldots, m-1$. Each pair $\left(V_{i}, W_{i}\right)$ consists of a displacement mode $V_{i}$ with the corresponding change in pressure distribution $W_{i}$. From now on, the superscript $n+1$ is dropped for clarity as all variables are at $t^{n+1}$. However, the $V_{i}$ and $W_{i}$ can contain data from previous time steps. During the first time step $(n=0)$ and when no data from the previous time steps are used $(l=0), m=1$ if $k=1$ and then no pair $\left(V_{i}, W_{i}\right)$ is available. We explain this exceptional case $(m=1)$ first, before the general case $(m>1)$.

- No pair $\left(\mathbf{V}_{\mathbf{i}}, \mathbf{W}_{\mathbf{i}}\right)$ is available $(\mathbf{m}=1)$

No displacements or changes in pressure distribution can be computed.

We use Newton's method to calculate $X_{2}$ with $P=P_{1}$. The Newton iterations are indicated with a second subscript $j+1(j=0, \ldots)$ and start from $X_{2,0}=X_{1}$.

$$
X_{2, j+1}=X_{2, j}-\left(\frac{\partial S}{\partial X}\right)_{X_{2, j}, P_{1}}^{-1} \cdot S\left(X_{2, j}, P_{1}\right)
$$

$X$ is underrelaxed with factor $\omega$ as explicit stepping for the pressure is employed in Eq. (24).

$$
X_{2} \equiv \omega X_{2}+(1-\omega) X_{1}
$$

For the examples in this paper, $\omega=0.001$ is used. The pressure distribution on the liquid side of the interface is then calculated with the flow solver.

$$
P_{2}=F\left(X_{2}\right)
$$




\section{- At least one pair $\left(\mathrm{V}_{\mathrm{i}}, \mathrm{W}_{\mathrm{i}}\right)$ is available $(\mathrm{m}>1)$}

If $m>1$, the displacement modes $V_{i}$ and the corresponding changes in pressure distribution $W_{i}$, with $i=1, \ldots, m-1$, are known. In order to have a reduced order model of the flow solver, we want to decompose a new displacement $\Delta X$ as a linear combination of the $V_{i}$.

$$
\Delta X \approx V \cdot \alpha
$$

with

$$
\begin{aligned}
\Delta X & =X-X_{k} \\
V & =\left[\begin{array}{llll}
V_{1} & V_{2} & \ldots & V_{m-1}
\end{array}\right] \\
\alpha & =\left[\begin{array}{llll}
\alpha_{1} & \alpha_{2} & \ldots & \alpha_{m-1}
\end{array}\right]^{\mathrm{T}}
\end{aligned}
$$

This is an overdetermined set of equations as the dimension of $X$ is larger than $m-1$. To minimize the Euclidian norm squared of $\Delta X-V \cdot \alpha$, we use linear least squares.

$$
\alpha=\left(V^{\mathrm{T}} \cdot V\right)^{-1} \cdot V^{\mathrm{T}} \cdot \Delta X
$$

The change of $P$ corresponding to $\Delta X$ can be estimated as

$$
\Delta P=W \cdot \alpha
$$

with

$$
\begin{aligned}
\Delta P & =P-P_{k} \\
W & =\left[\begin{array}{llll}
W_{1} & W_{2} & \ldots & W_{m-1}
\end{array}\right]
\end{aligned}
$$

We define $A=W \cdot\left(V^{\mathrm{T}} \cdot V\right)^{-1} \cdot V^{\mathrm{T}}$, so Eq. (29a) becomes

$$
\Delta P=A \cdot \Delta X
$$

The reduced order model for $P$ is given by 


$$
\begin{aligned}
\hat{P} & =P_{k}+\Delta P \\
& =P_{k}+A \cdot\left(X-X_{k}\right) \\
& =\hat{F}(X) .
\end{aligned}
$$

To distinguish between distributions of the pressure on the liquid side of the interface coming from the reduced order model and distributions from the flow solver, a hat is used for those from the reduced order model. The Jacobian of this reduced order model is

$$
\frac{\mathrm{d} \hat{P}}{\mathrm{~d} X}=\frac{\mathrm{d} \hat{F}(X)}{\mathrm{d} X}=A
$$

The reduced order model is substituted in Eq. (14). So, Eq. (14) can be solved with Newton's method, with $X_{k+1,0}=X_{k}$. After every Newton iteration, $P$ is updated with the reduced order model.

$$
\begin{aligned}
X_{k+1, j+1}= & X_{k+1, j} \\
& -\left(\frac{\partial S}{\partial X}+\frac{\partial S}{\partial P} \frac{\mathrm{d} \hat{P}}{\mathrm{~d} X}\right)_{X_{k+1, j}, \hat{P}_{k+1, j}}^{-1} \cdot S\left(X_{k+1, j}, \hat{P}_{k+1, j}\right) \\
\hat{P}_{k+1, j+1}= & P_{k}+\frac{\mathrm{d} \hat{P}}{\mathrm{~d} X} \cdot\left(X_{k+1, j+1}-X_{k}\right)
\end{aligned}
$$

When the Newton iterations have converged, the flow solver is used to obtain $P_{k+1}$.

$$
P_{k+1}=F\left(X_{k+1}\right)
$$

The maximal node displacement from $X_{k}$ to $X_{k+1}$ is monitored. If the displacement has decreased enough, the time step has converged. Otherwise, another coupling iteration has to be performed. The convergence criteria we used in the examples are given in the following section. 


\section{Results and discussion}

\subsection{Oscillating droplet}

With the implicitly coupled partitioned solvers, the oscillation of a water droplet in air is simulated. An unstructured triangular grid used to calculate the water flow is shown in Figure 4.

The horizontal and vertical edges of the grid are the axes of the coordinate system. The problem is considered to be axisymmetric around the horizontal axis, but also symmetric around the plane normal to the horizontal axis through the origin. Gravity is neglected. The initial shape of the droplet of water at rest is the second spherical harmonic. The radius $R$ at the beginning of the simulation is given as a function of the polar angle $\theta$.

$$
R(\theta)=R_{0} \cdot\left[1+a_{2,0} \cdot \mathcal{P}_{2}(\cos \theta)\right]
$$

$a_{2,0}$ is the initial amplitude of the second spherical harmonic and $\mathcal{P}_{2}$ the second Legendre polynomial. The oscillation amplitude reduces due to viscosity and finally the droplet becomes a sphere with equilibrium radius $R_{0}$.

The physical properties of water (subscript 1) and the water-air interface used for the simulations are given in Table 1. The flow solver for the water is a segregated incompressible solver with SIMPLE pressure-velocity coupling and a first order implicit unsteady formulation. A second order upwind scheme is used for the discretization of the momentum equation and the pressure at the faces is interpolated from the cell centered values with momentum equation coefficients. Local remeshing preserves the quality of the mesh: large cells are split, small cells are merged and the skewness of the cells is automatically 
limited.

The liquid domain does not have a boundary condition to set the absolute pressure, so the pressure coming from the flow solver is determined up to a constant. The physically correct value of that constant is calculated by minimizing the root mean square value of $S$ after the call to the flow solver. The constant to set the absolute pressure level is an additional unknown, which can be viewed as a Lagrange multiplier for the constant volume constraint which is added to the structural solver.

The angular frequency and the time constant of the damping of the small amplitude oscillation can be compared with the linear, irrotational approximation for low viscosity fluids by Lamb [21], who analyzed the droplet oscillation as an infinite sum of spherical harmonics. In this theory, Eq. (2) is used on the interface. The angular frequency $\hat{\omega}_{i}$ of the $i^{\text {th }}$ mode of an oscillating liquid droplet is given by Eq. (36a) as a function of the undamped angular frequency $\omega_{i}$ and the time constant $\tau_{i}$ :

$$
\hat{\omega}_{i}=\omega_{i} \sqrt{1-\left(\omega_{i} \tau_{i}\right)^{-2}}
$$

with:

$$
\begin{aligned}
\omega_{i}^{2} & =\frac{\sigma}{\rho R_{0}{ }^{3}} i(i-1)(i+2) \\
\tau_{i} & =\frac{\rho R_{0}{ }^{2}}{\mu(i-1)(2 i+1)} .
\end{aligned}
$$

The second mode is triggered by the initial shape, with an initial amplitude of $a_{2,0}=0.01$. According to Lamb's theory, the amplitude of the oscillation is

$$
a_{2}(t)=a_{2,0} \exp \left(-t / \tau_{2}\right) \cos \left(\hat{\omega}_{2} t\right)
$$


A time step dependence study has been performed, with $N=21$ nodes on the interface and $R_{0}=1 \mathrm{~mm}$. The number of nodes on the interface has little effect on $\hat{\omega}_{2}$ and $\tau_{2}$ as shown in Table 2. Consequently, there are $N=21$ nodes on the interface in all simulations of the time step dependence study. The time step $\Delta t$ varies from $\Delta T=0.00035 \mathrm{~s}$ to $\Delta T / 128$. The results of the time step dependence study for $\hat{\omega}_{2}$ and $\tau_{2}$ are given in Table 3 . The values for $\hat{\omega}_{2}$ and $\tau_{2}$ are calculated during the second period of the oscillation to minimize the effect of the initial condition where the fluid is at rest. The Richardson extrapolation $\gamma_{\text {extr }}$ of a quantity $\gamma$ is calculated with the values of the 3 simulations with the smallest time steps $(\gamma(\Delta T / 32), \gamma(\Delta T / 64)$ and $\gamma(\Delta T / 128))$ :

$$
\begin{aligned}
\gamma_{\text {extr }} & =\gamma(\Delta T / 64)+\frac{\gamma(\Delta T / 128)-\gamma(\Delta T / 64)}{1-2^{-\alpha}} \\
\alpha & =\frac{\ln \left(\frac{\gamma(\Delta T / 32)-\gamma(\Delta T / 64)}{\gamma(\Delta T / 64)-\gamma(\Delta T / 128)}\right)}{\ln (2)} .
\end{aligned}
$$

The Error given in Table 3 is the relative difference with the Richardson extrapolation.

Time constant $\tau_{2}$ is calculated as

$$
\tau_{2}=\frac{t_{\max 2}-t_{\max 1}}{\ln \left(\frac{a_{2, \max 1}}{a_{2, \max 2}}\right)} .
$$

with $t_{\max }$ the time when a maximum occurs and $a_{2 \text {,max }}$ the amplitude at the maximum. The parameters on the right hand side of Eq. (40) are indicated on Figure 5. Once $\tau_{2}$ has been calculated for a simulation, we express that the time derivative of the amplitude is zero in a maximum which gives us an equation in $\hat{\omega}_{2}$.

$$
\cos \left(\hat{\omega}_{2} t_{\max }\right)=\frac{1}{\sqrt{1+\left(\tau_{2} \hat{\omega}_{2}\right)^{-2}}} .
$$


The amplitude of the oscillation is shown in Figure 5 for the different time step sizes, together with Lamb's theory and the Richardson extrapolation. The extrapolation is close to Lamb's theory. The time convergence is however slow due to the first order time accuracy of the flow solver. This once again emphasizes the need for higher order time accuracy on moving grids [7].

The number of coupling iterations required to bring the maximal node displacement below $1 / 10^{8}$ of the droplet radius each time step of the simulation with $\Delta t=\Delta T / 64$ is given in Figure 6(a), with an average of 9.8. The convergence behaviour of a time step with the minimal, average and maximal number of coupling iterations is shown in Figure 6(b). The minimal, average and maximal number of coupling iterations required to bring the maximal node displacement below $1 / 10^{8}$ of the droplet radius is shown in Table 4 for the simulations of the time step dependence study. Data from the previous time step are used to improve the reduced order model $(l=1)$. The number of coupling iterations increases with the decreasing time step: due to inertia, a given error on the displacement of the interface results in a larger error on the pressure on the liquid side of the interface when the time step is smaller.

Table 5 shows the number of coupling iterations for the same simulation as Table 4, but without using data from the previous time step in the reduced order model. If the number of coupling iterations in Table 5 is compared with that in Table 4, it is shown that using the data from the previous time step reduces the number of coupling iterations. If the data of the two previous time steps are used $(l=2)$, the coupling iterations do not always converge for these examples. We therefore do not present results for $l=2$. We only mention that in the simulation with time step $\Delta t=\Delta T / 128$ and $l=2$, the average number of coupling iterations in the converged time steps decreases by approximately 
$10 \%$ with respect to $l=1$. The number of coupling iterations is independent of the number of nodes on the interface, as can be seen from Table 6 .

\subsection{Bubble growth and detachment from a vertical needle}

The second application of the model is the growth and detachment of an air bubble from a vertical needle with inner radius $r$, submerged in quiescent water in the gravitational field. A specific case already studied experimentally by Longuet-Higgins et al. [22] and numerically by Og̃uz et al. [27], has been simulated. The scheme of the geometry is given in Figure 7.

The air mass flow rate $\dot{m}$ through the needle is modelled as done by Og̃uz et al. [27].

$$
\dot{m}=\frac{\pi}{16} \frac{r^{4}}{L \mu_{1}} \frac{p_{C}^{2}-p_{1}^{2}}{\mathcal{R} T}
$$

with $p_{C}$ the pressure in the device delivering the mass flow, $\mathcal{R}$ the specific gas constant of air $\left(287 \frac{\mathrm{J}}{\mathrm{kgK}}\right)$ and $T$ the temperature which is modelled constant at $297 \mathrm{~K} . p_{1}$ is the pressure inside the bubble and $\mu_{1}$ is the dynamic viscosity of air. $L$ is the effective length of the needle: constrictions are converted into an equivalent needle length.

Eq. (42) is integrated implicitly in time, to avoid time step restrictions.

$$
m^{n+1}=m^{n}+\frac{\pi}{16} \frac{r^{4}}{L \mu_{1}} \frac{p_{C}^{2}-p_{1}^{n+1^{2}}}{\mathcal{R} T} \cdot \Delta t^{n+1}
$$

The air pressure is calculated with the ideal gas law.

$$
p_{1}^{n+1}=\frac{m^{n+1} \mathcal{R} T}{\mathcal{V}^{n+1}}
$$


with $\mathcal{V}$ the volume of the bubble, calculated from the position of the interface nodes. Eqs. (43) and (44) are a set of two equations, resulting in a quadratic equation for $m^{n+1}$. The physically correct solution for $m^{n+1}$ is selected and then substituted in Eq. (44) to obtain $p_{1}^{n+1}$

The pressure at the top of the computational domain is modelled as a pressure outlet with a uniform pressure. To compensate for the change of the bubble volume, the pressure at the outlet is adjusted with

$$
\frac{\mathcal{V}^{n+1}-\mathcal{V}^{0}}{\pi \cdot R^{2}} \cdot \rho_{2} \cdot g
$$

with $g$ the gravitational acceleration and $R$ the radius of the reservoir. The interface is modelled as free slip wall. All other boundaries except for the top of the domain are no slip walls. The point where the interface touches the needle's top is fixed.

The bubble starts as half a sphere at the end of the needle. In this situation the radii of curvature are at their lowest value, so the air pressure $p_{1}$ in the bubble is maximal.

$$
p_{1}=p_{\infty}+\frac{2 \sigma}{r}
$$

with $p_{\infty}$ the stagnation pressure at the needle's tip on the water side of the interface. This situation can only be reached if $p_{C}>p_{\infty}+2 \sigma / r$. The pressure difference between the air in the bubble and the device delivering the mass flow is small in this situation and bubble growth is slow. The initial water velocity is set to zero. This initial condition causes a sudden but small oscillation of the position of the bubble's top above the needle, but the amplitude is within $0.1 \%$ of the needle radius after $0.003 \mathrm{~s}$ while the formation of a complete 
bubble takes $0.65 \mathrm{~s}$.

For this simulation the parameters stated in Table 7 are used [27], resulting in a volumetric growth rate around $200 \mathrm{~mm}^{3} / \mathrm{s}$. The settings for the flow solver are identical to those described in Section 4.1. The area of the bubble in a cross-section orthogonal to the needle's axis is monitored in the nodes on the interface close to the needle's outlet. When the smallest area is within $1 \%$ of the area of the needle's cross-section, the node closest to the axis is duplicated and both nodes are placed on the axis of symmetry. This way the interface is split in two parts.

To investigate the influence of the number of nodes on the interface and the time step, we performed a simulation with coarse, medium and fine resolution. The size of the first time step $\Delta t$, the initial number of nodes on the interface and the initial number of cells of the unstructured grid in the water are given in Table 8 for these 3 simulations. The number of nodes on the bubble interface is constant while the number of cells in the water varies slightly during the simulation.

The time step size is adapted at the onset of every time step to have a node displacement that is close to the maximal node displacement of the first time step.

$$
\Delta t^{n+1}=\left(1-\omega_{t}\right) \Delta t^{n}+\omega_{t} \frac{\max \left|X^{1}-X^{0}\right|}{\max \left|U^{n}\right|}
$$

with the relaxation factor $\omega_{t}$ set to 0.2 for this example. The time step is adapted because the dynamics of the bubble alter a lot during the simulation.

The shape of the bubble just before pinch off is shown in Figure 8. The position 
of the bubble's top above the needle $h_{t}$ at detachment is shown in Table 8 for the 3 simulations. From Table 8, it can be seen that the difference between the simulations is small. The position of the bubble's top $h_{t}$ above the needle as a function of time is shown in Figure 9. It can be seen that the results from the simulations with coarse, medium and fine resolution match.

Further results are derived from the simulation with medium resolution. The simulated bubble shapes are compared with the experimental results of LonguetHiggins et al. at 10 different moments and they show good agreement (Figure 10).

Figure 11(a) shows the number of coupling iterations needed to drop the maximal node displacement below $1 / 10^{3.3}$ of the needle radius for every time step, with an average of 10.29. As the time step decreases towards the detachment of the bubble in time step 705, the number of coupling iterations increases. After detachment of the bubble, the time step increases and so the number of coupling iterations decreases again. Time steps with a high number of coupling iterations build up a good reduced order model and the subsequent time step only needs few coupling iterations. As only data of the previous time step are used, the following time step starts with a poor reduced order model and requires a lot of coupling iterations. The number of coupling iterations oscillates from high to low during the time steps, as can be seen clearly on Figure 11(a). Again, when the data from the two previous time steps are used, the coupling iterations do not always converge and therefore $l=2$ is not used here. As the number of coupling iterations is very high near the detachment of the bubble, there are so many displacement modes and corresponding changes in pressure distribution that it becomes very likely that the data will contradict itself if data from several time steps are used, which leads to divergence of 
the coupling iterations. Figure 11(b) shows the convergence behaviour of the coupling iterations for the fastest, an average and the slowest converging time step.

\section{Conclusions}

In this paper, the interface between a gas and a liquid is represented by a zero thickness structure. We calculate the position of the interface with a structural solver and the pressure on the liquid side of the interface with a flow solver. The focus is on the implicit coupling technique between the accessible structural solver and the black box flow solver, without adding compressibility to the liquid. The structural solver uses a reduced order model of the flow solver, generated during the coupling iterations of the time step, to predict the pressure on the liquid side of the interface during the calculation of a new interface position. We presented a grid and time step dependence study for two axisymmetric problems: the oscillation of a liquid droplet and the growth and detachment of an air bubble from the outlet of a vertical needle, submerged in quiescent water in the gravitational field. The simulations correspond well with analytical theories and experiments and the computational cost of the coupling is affordable. The sharp representation of the interface gives accurate results even with a coarse grid. This coupling technique can be applied to other Fluid-Structure Interaction problems with one accessible solver. The technique is independent of the flow solver code used to demonstrate the coupling. 


\section{Acknowledgments}

The authors acknowledge the funding of Joris Degroote and Peter Bruggeman by a Ph.D. fellowship of the Research Foundation - Flanders (FWO).

\section{References}

[1] Bathe KJ, Zhang H, Ji SH. Finite element analysis of fluid flows fully coupled with structural interactions. Comput Struct 1999;72:1-16.

[2] Brackbill J, Kothe D, Zemach C. A continuum method for modelling surface tension. J Comp Phys 1992;100:335-354.

[3] Causin P, Gerbeau JF, Nobile F. Added-mass effect in the design of partitioned algorithms for fluid-structure problems. Comput Meth Appl Mech Eng 2005;194:4506-4527.

[4] Deng ZT, Jeng SM. Numerical simulation of droplet deformation in convective flows. AIAA Journal 1992;30:1290-1297.

[5] Deparis S, Discacciati M, Fourestey G, Quarteroni A. Fluid-structure algorithms based on Steklov-Poincaré operators. Comput Meth Appl Mech Eng 2006;195:5797-5812.

[6] Donea J, Huerta A, Ponthot JP, Rodriguez-Ferran A. Encyclopedia of Computational Mechanics, Chapter 14 Arbitrary Lagrangian-Eulerian Methods. John Wiley \& Sons, Ltd.; 2004.

[7] Farhat C, Geuzaine P. Design and analysis of robust ALE time-integrators for the solution of unsteady flow problems on moving grids. Comput Meth Appl Mech Eng 2004;193:4073-4095. 
[8] Farhat C, Lesoinne M, LeTallec P. Load and motion transfer algorithms for fluid-structure interaction problems with non-matching discrete interfaces: Momentum and energy conservation, optimal discretization and application to aeroelasticity. Comput Meth Appl Mech Eng 1998;157:95-114.

[9] Fernández M, Moubachir M. A Newton method using exact Jacobians for solving fluid-structure coupling. Comput Struct 2005;83:127-142.

[10] Frisch U, Hasslacher B, Pomeau Y. Lattice-gas automata for the Navier-Stokes equations. Phys Rev Lett 1986;56:1505-1508.

[11] Gerbeau JF, Vidrascu M. A quasi-Newton algorithm based on a reduced model for fluid-structure interaction problems in blood flows. ESAIM Math Model Numer Anal 2003;37:631-647.

[12] Glimm J, Grove JW, Li XL, Oh W, Sharp DH. A critical analysis of Rayleigh-Taylor growth rates. J Comp Phys 2001;169:652-677.

[13] Harlow FH, Welch JE. Numerical calculation of time-dependent viscous incompressible flow. Phys Fluid 1965;8:2182-2189.

[14] Heil, M. An efficient solver for the fully coupled solution of large-displacement fluid-structure interaction problems. Comput Meth Appl Mech Eng 2004;193:123.

[15] Hirt C, Nichols B. Volume of fluid method for the dynamics of free boundaries. J Comp Phys 1981;39:201-225.

[16] Hnat JG, Buckmaster JD. Spherical cap bubbles and skirt formation. Phys Fluid 1976;19:182-194.

[17] Hou TY, Lowengrub JS, Shelley MJ. Boundary integral methods for multicomponent fluids and multiphase materials. J Comp Phys 2001;169:302362. 
[18] Hubner B, Walhorn E, Dinkler D. A monolithic approach to fluid-structure interaction using space-time finite elements. Comput Meth Appl Mech Eng 2004;193:2087-2104.

[19] Kleefsman KMT, Fekken G, Veldman AEP, Iwanowski B, Buchner B. A Volume-of-Fluid based simulation method for wave impact problems. J Comp Phys 2005;206:363-393.

[20] Lafaurie B, Nardone D, Scardovelli R, Zaleski S, Zanetti G. Modelling merging and fragmentation in multiphase flows with surfer. J Comp Phys 1994;113:134147.

[21] Lamb H. Hydrodynamics. Cambridge University Press, 6 ed.; 1932.

[22] Longuet-Higgins M, Kerman B, Lunde K. The release of air bubbles from an underwater nozzle. J Fluid Mech 1991;230:365-390.

[23] Matthies HG, Niekamp R, Steindorf J. Algorithms for strong coupling procedures. Comput Methods Appl Mech Eng 2006;195:2028-2049.

[24] Matthies HG, Steindorf J. Partitioned strong coupling algorithms for FluidStructure Interaction. Comput Struct 2003;81:805-812.

[25] Michler C, van Brummelen E, de Borst R. Error-amplification analysis of subiteration-preconditioned GMRES for fluid-structure interaction. Comput Methods Appl Mech Eng 2006;195:2124-2148.

[26] Mok DP, Wall WA, Ramm E. Accelerated iterative substructuring schemes for instationary fluid-structure interaction. In: Bathe K, editors. Computational Fluid and Solid Mechanics. Elsevier; 2001. p.1325-1328

[27] Og̃uz H, Prosperetti A. Dynamics of bubble growth and detachment from a needle. J Fluid Mech 1993;257:111-145. 
[28] Oñate E, Idelsohn SR, Del Pin F, Aubry R. The particle finite element method. An overview. Int J Comput Meth 2004;1:267-307.

[29] Osher S, Fedkiw RP. Level set methods: an overview and some recent results. J Comp Phys 2001;169:463-502.

[30] Piperno S. Explicit/implicit fluid/structure staggered procedures with a structural predictor and fluid subcycling for 2D inviscid aeroelastic simulations. Int J Numer Meth Fluid 1997;25:1207-1226.

[31] Piperno S, Farhat C. Partitioned procedures for the transient solution of coupled aeroelastic problems - Part II: energy transfer analysis and three-dimensional applications. Comput Meth Appl Mech Eng 2001;190:3147-3170.

[32] Piperno S, Farhat C, Larrouturou B. Partitioned procedures for the transient solution of coupled aeroelastic problems. I. model problem, theory and twodimensional application. Comput Meth Appl Mech Eng 1995;124:79-112.

[33] Pozrikidis C. Interfacial Dynamics for Stokes Flow. J Comp Phys 2001;169:250301.

[34] Rider WJ, Kothe DB. Reconstructing Volume Tracking. J Comp Phys $1998 ; 141: 112-152$.

[35] Rothman DH, Zaleski S. Lattice-gas models of phase separation: interfaces, phase transitions and multiphase flow. Rev Mod Phys 1994;66:1417-1479.

[36] Ryskin G, Leal LG. Numerical solution of free-boundary problems in fluid mechanics. Part 2. Bouyancy-driven motion of a gas bubble through a quiescent liquid. J Fluid Mech 1984;148:19-35.

[37] Steindorf J. Partitionierte Verfahren für Probleme der Fluid-Struktur Wechselwirkung. Doctoral thesis, Technische Universität Braunschweig, Brunswick; 2002. 
[38] Sussman M, Fatemi E, Smereka P, Osher S. An improved level set method for incompressible two-phase flows. Comput Fluid 1998;27:663-680.

[39] Tezduyar TE. Methods for computation of moving boundaries and interfaces. In: European Congress on Compuational Methods in Applied Sciences and Engineering, ECCOMAS 2004. Jyväskylä, Finland; 2004.

[40] Tomé M, McKee S. Gensmac: A computational marker and cell method for free surface flows in general domains. J Comp Phys 1994;110:171-186.

[41] Tryggvason G. Numerical simulations of the Rayleigh-Taylor instability. J Comp Phys 1988;75:253-282.

[42] Tryggvason G, Bunner B, Esmaeeli A, Juric D, Al-Rawahi N, Tauber W, Han J, Nas S, Jan YJ. A front-tracking method for the computations of multiphase flow. J Comp Phys 2001;169:708-759.

[43] Vierendeels J. Implicit coupling of partitioned fluid-structure interaction solvers using a reduced order model. In: Proc. of the 35th AIAA Fluid Dynamics Conference and Exhibit, vol. AIAA-2005-5135. Toronto, Ontario, Canada; 2005.

[44] Vierendeels J, Dick E, Verdonck P. Hydrodynamics of color M-mode Doppler flow wave propagation velocity V(p): A computer study. J Am Soc Echocardiogr 2002;15:219-224.

[45] Vierendeels J, Riemslagh K, Dick E, Verdonck P. Computer simulation of intraventricular flow and pressure gradients during diastole. J Biomech Eng 2000;122:667-674.

[46] Wall WA, Genkinger S, Ramm E. A strong coupling partitioned approach for fluid-structure interaction with free surfaces. Comput Fluid 2007;36:169-183.

[47] Willcox K, Peraire J, White J. An Arnoldi Approach for Generation of ReducedOrder Models for Turbomachinery. Comput Fluid 2002;31:369-389. 
[48] Yabe T, Xiao F, Utsumi T. The constrained interpolation profile method for multiphase analysis. J Comp Phys 2001;169:556-593.

[49] Zufiria JA. Vortex-in-cell simulation of bubble competition in a Rayleigh-Taylor instability. Phys Fluid 1988;31:3199-3212.

[50] van Zuijlen AH, Bijl H. Implicit and explicit higher order time integration schemes for structural dynamics and fluid-structure interaction computations. Comput Struct 2005;83:93-105. 
(a)

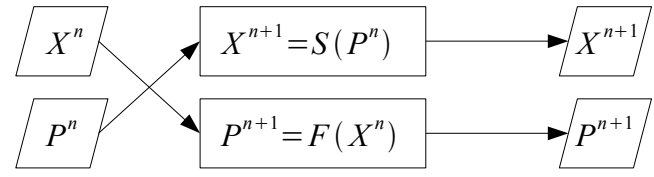

(b)

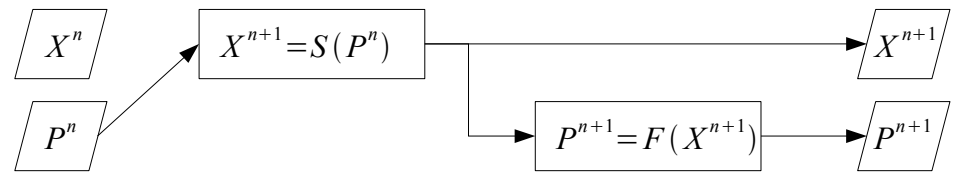

(c)

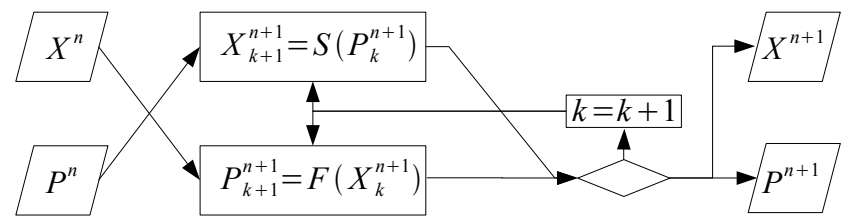

(d)

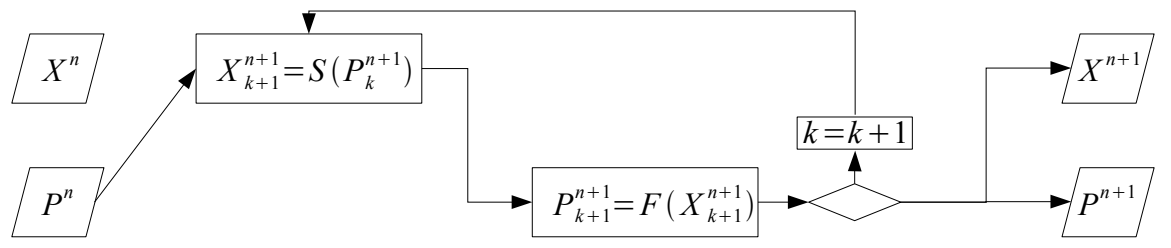

Fig. 1. Coupling schemes for a flow solver $F$ and a structural solver $S$. $X$ is the position of the interface, $P$ is the pressure on the interface. (a) One parallel call to the solvers, (b) one serial call, (c) multiple parallel calls and (d) multiple serial calls. The superscript $n+1$ indicates the current time step and the subscript $k+1$ denotes the coupling iteration. $k$ starts from 0 and $X_{0}^{n+1}=X^{n}, P_{0}^{n+1}=P^{n}$. 


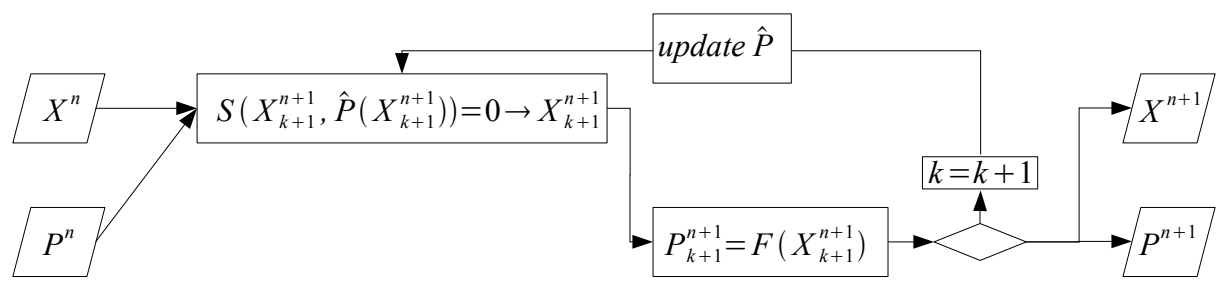

Fig. 2. Multiple serial calls to the solvers with implicit stepping of the pressure with a reduced order model for the flow solver inside the structural solver. The superscript $n+1$ indicates the current time step and the subscript $k+1$ denotes the coupling iteration. $k$ starts from 0 and $X_{0}^{n+1}=X^{n}, P_{0}^{n+1}=P^{n}$. 


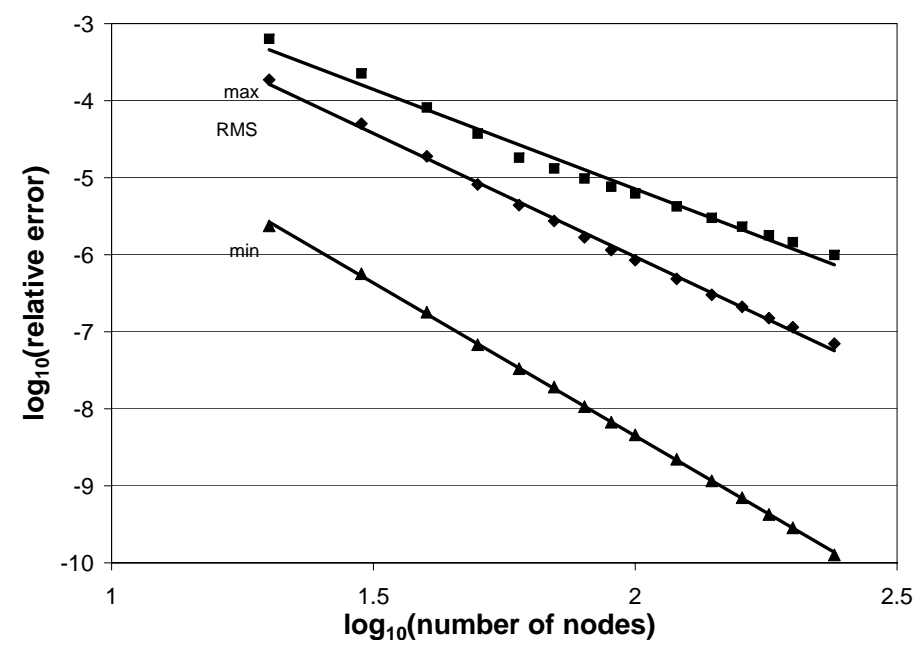

Fig. 3. Convergence of the curvature calculation for half an ellipsoid with semi-axes of $1.5 \mathrm{E}-3 \mathrm{~m}$ and $1.0 \mathrm{E}-3 \mathrm{~m}$. The maximal, minimal and root mean square value of the relative error over the surface are shown as a function of the number of nodes. The relative error is the absolute value of the difference between the numerical and analytical curvature, divided by the inverse of the long semi-axis. 


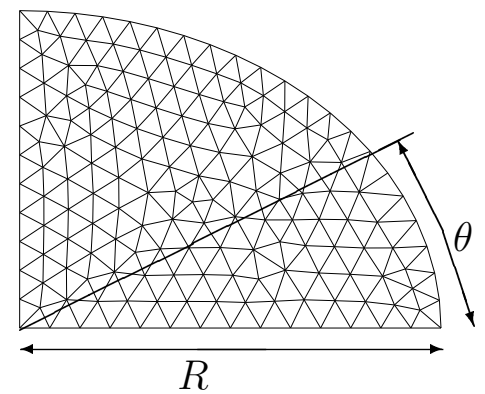

Fig. 4. An unstructured triangular grid used to calculate the flow in the water during the simulation of an oscillating droplet. The radius $R$ and the polar angle $\theta$ used in Eq. (35) are indicated. 


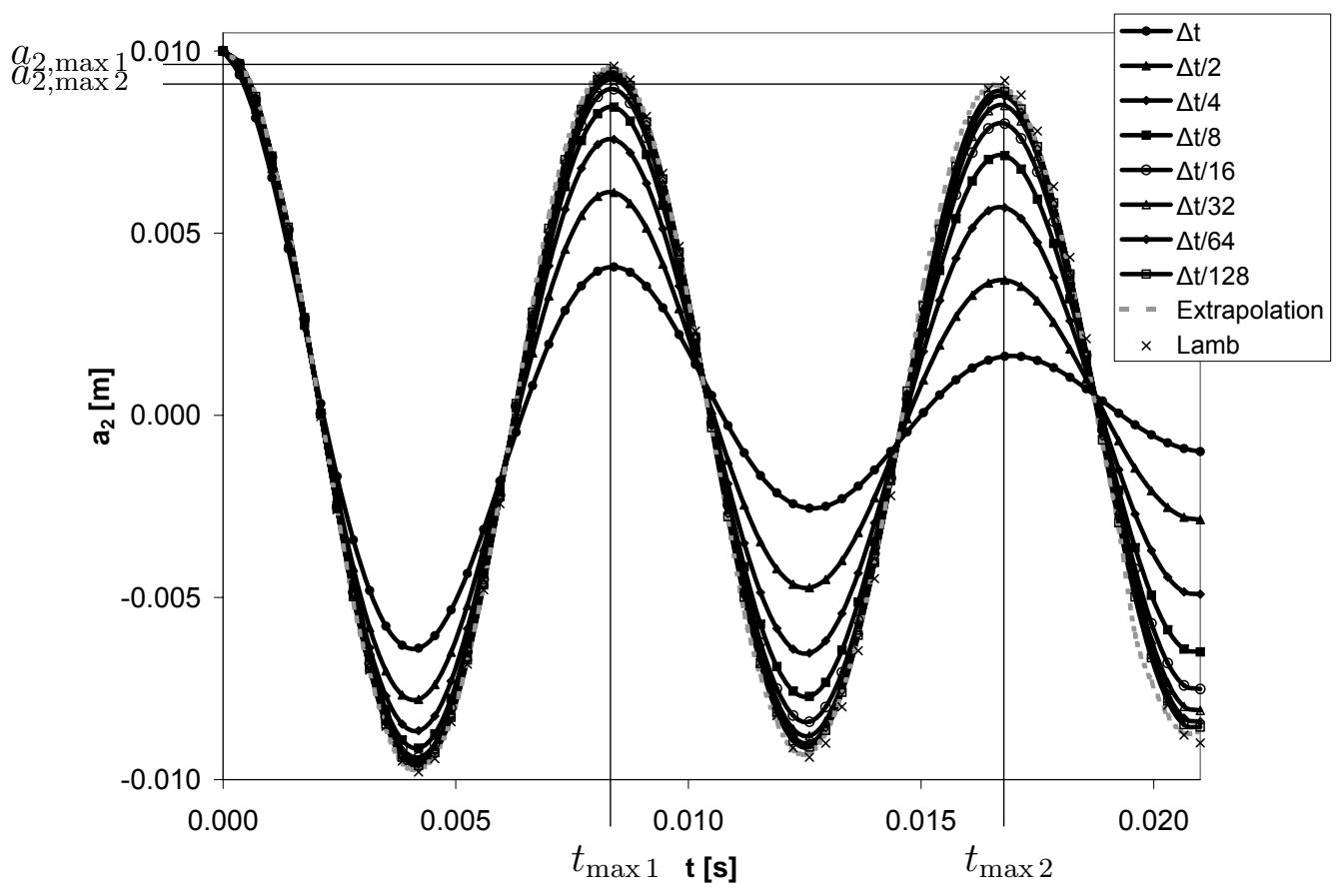

Fig. 5. Oscillation amplitude $a_{2}$ as a function of time for the small amplitude oscillation of a water droplet with time steps $\Delta t=\Delta T$ to $\Delta t=\Delta T / 128$, with $\Delta T=0.00035 \mathrm{~s}$, together with a Richardson extrapolation and Lamb's theory. The number of nodes on the interface is $N=21$ in all simulations. 


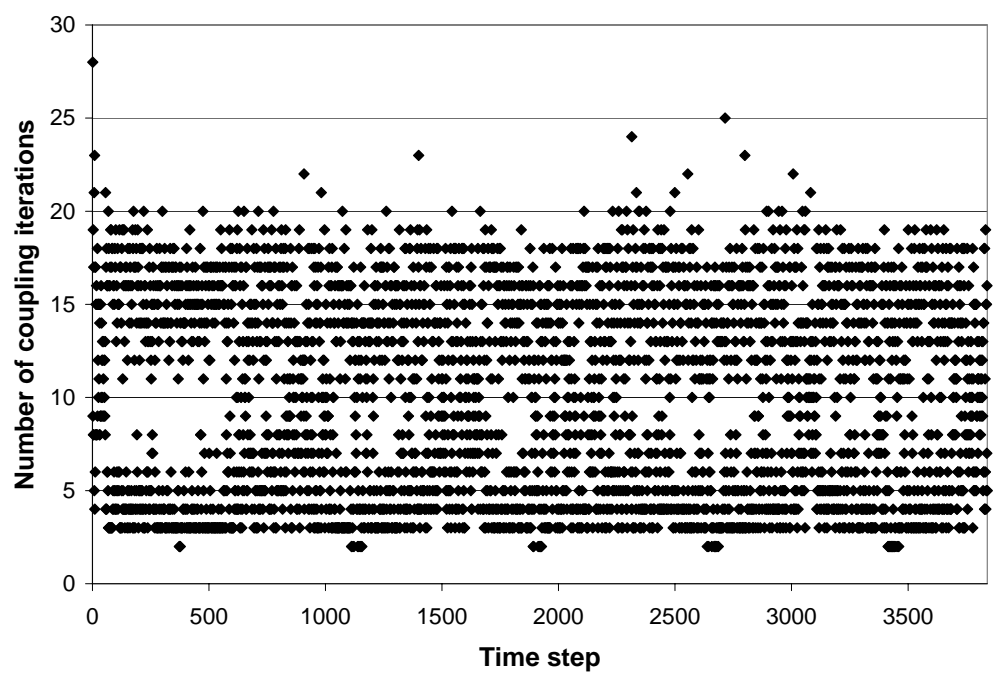

(a)

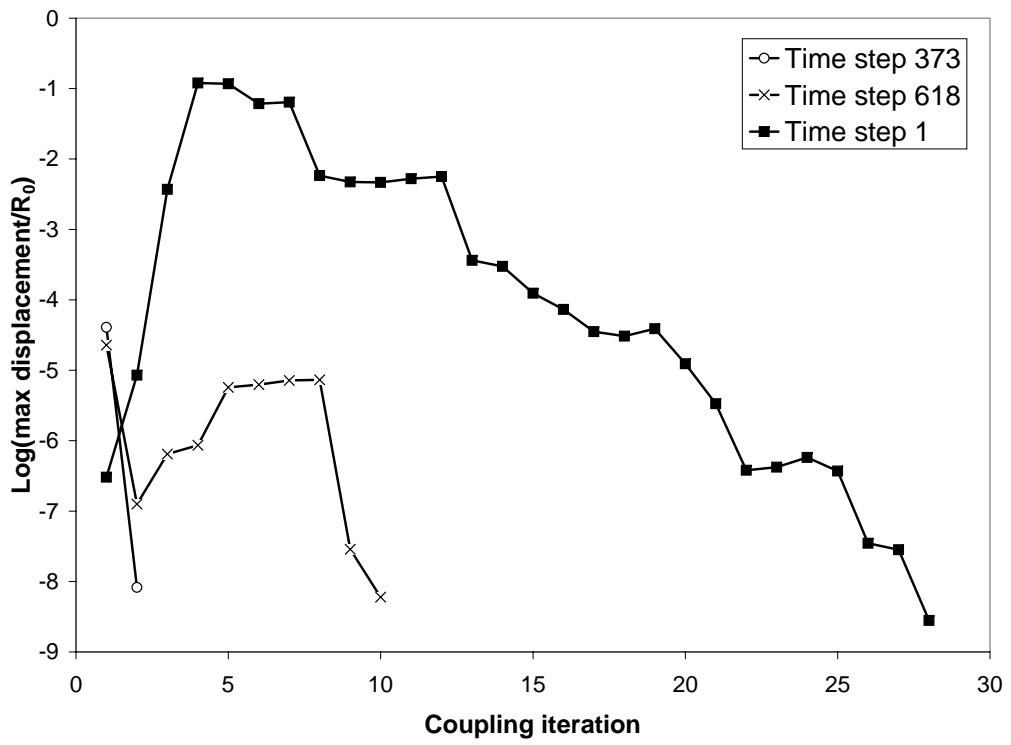

(b)

Fig. 6. (a) The number of coupling iterations required to bring the maximal node displacement below $1 / 10^{8}$ of the droplet radius each time step of the oscillation of a water droplet with $N=21$ nodes on the interface and time step $\Delta t=\Delta T / 64$ and (b) the convergence behaviour of the coupling iterations for time step 373 (2 iterations), 618 (10 iterations) and 1 (28 iterations). 


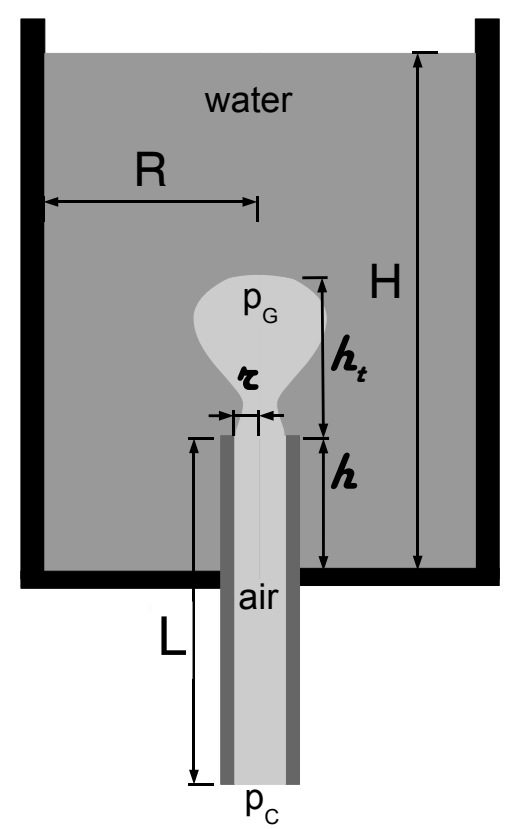

Fig. 7. Scheme of the geometry used for the simulation of bubble growth and detachment. $r=0.002 \mathrm{~m}, R=0.020 \mathrm{~m}, h=0.040 \mathrm{~m}, H=0.100 \mathrm{~m}$ and $L=128 \mathrm{~m}$. The constriction in the needle present in the experimental setup has been converted into an equivalent length of straight needle in the simulation. 


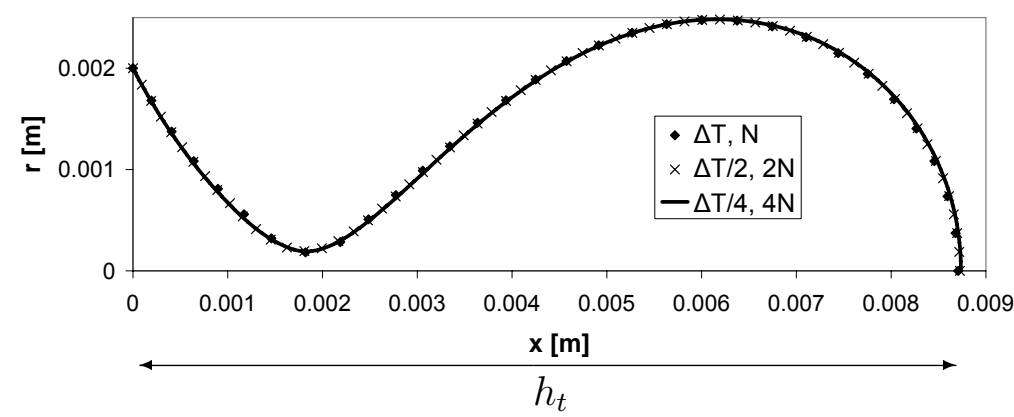

Fig. 8. Bubble shape just before pinch off for the coarse, medium and fine simulation, with $\Delta T=0.005 \mathrm{~s}$ and $N=32$. The position of the bubble's top above the needle $h_{t}$ is given in Table 8 . 


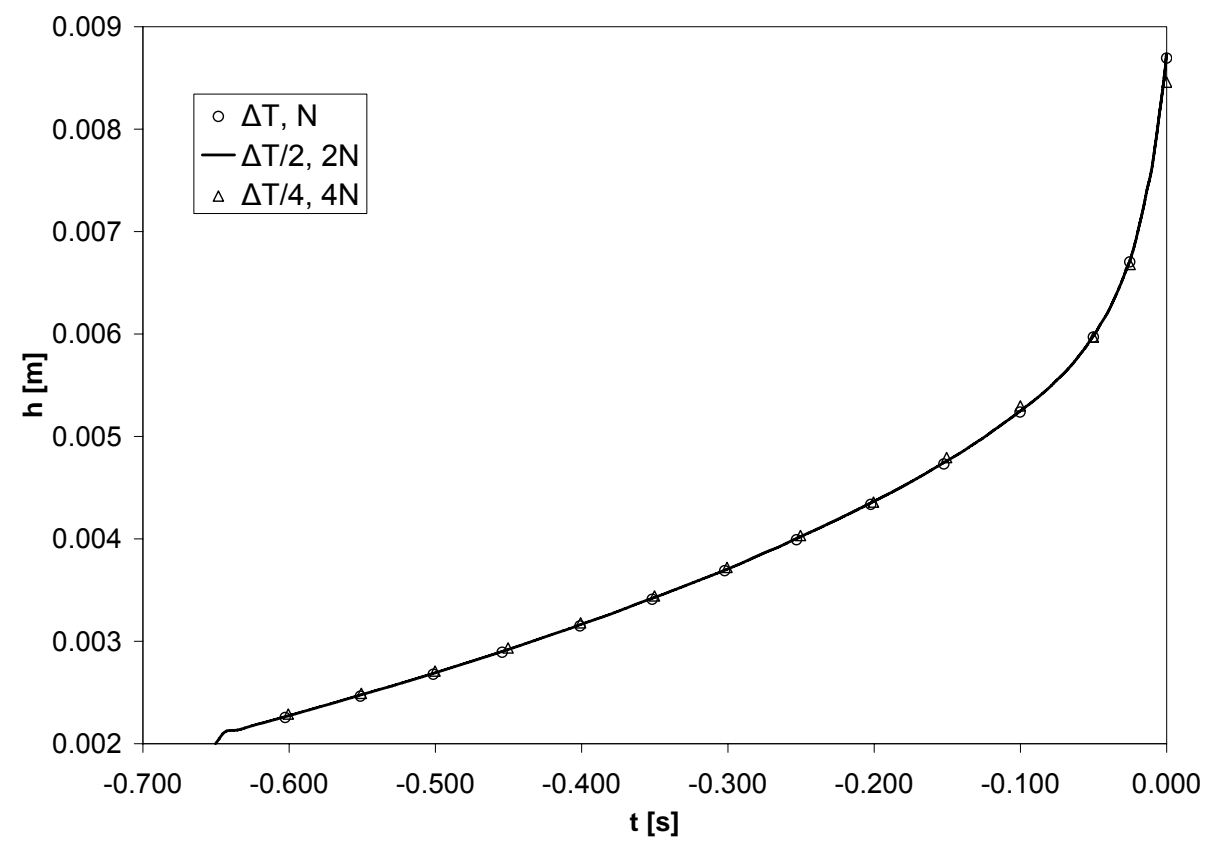

Fig. 9. The position of the bubble's top above the needle $h_{t}$ as a function of time for the coarse, medium and fine simulation, with $\Delta T=0.005 \mathrm{~s}$ and $N=32$. 

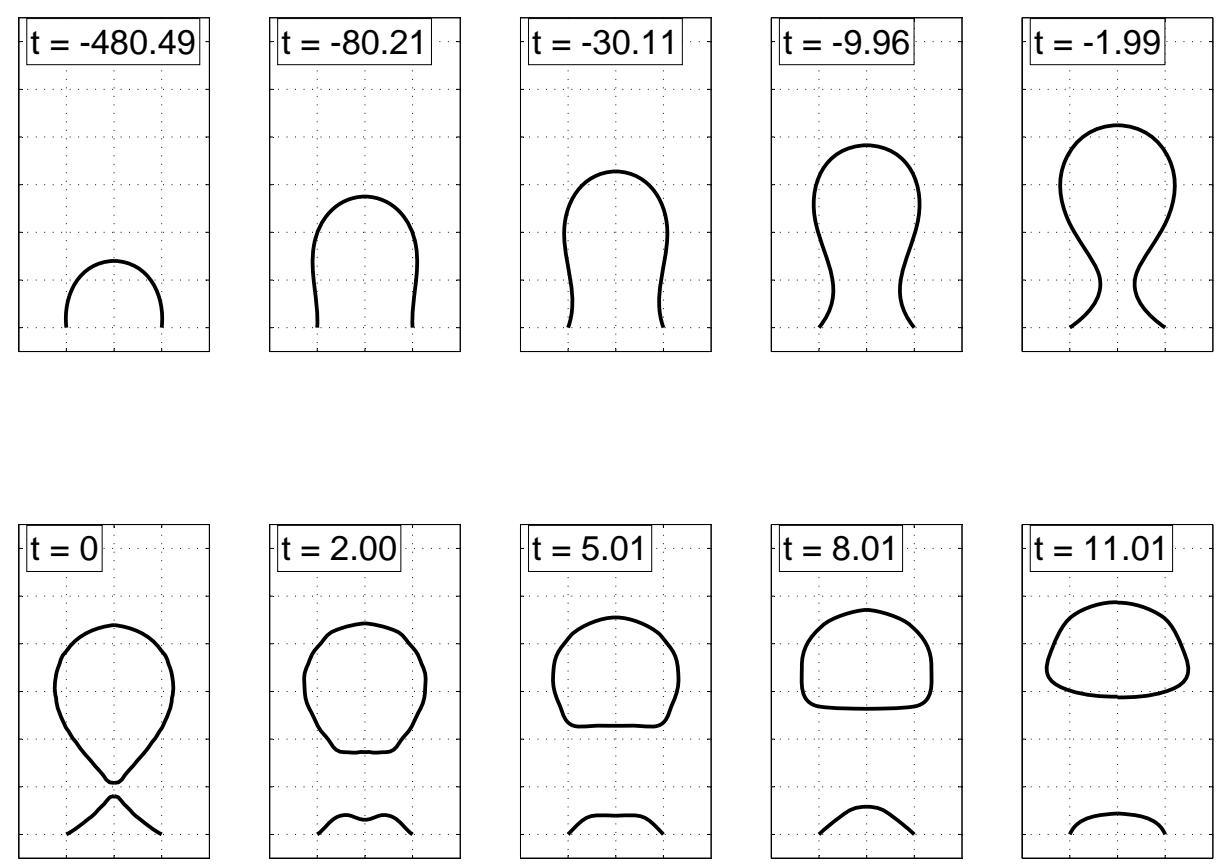

(a)
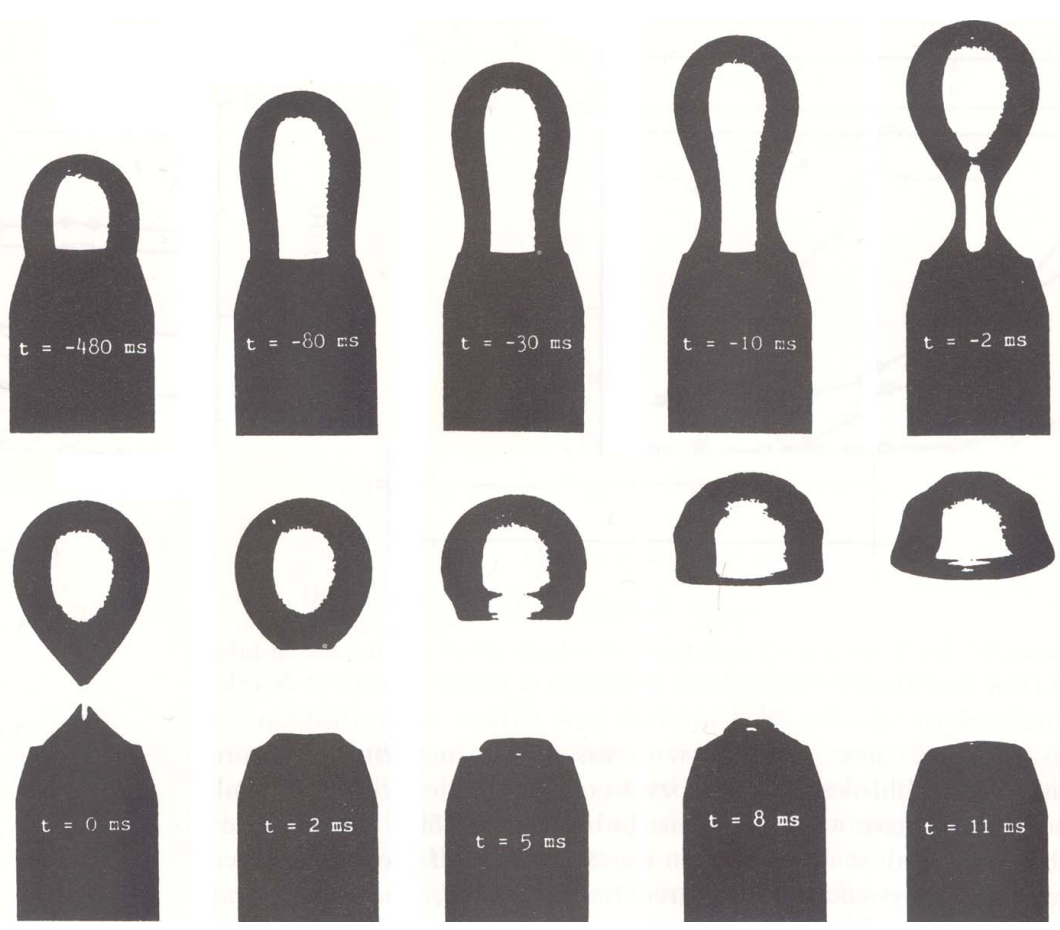

(b)

Fig. 10. Shape of the bubble at 10 different moments. Time $t$ in ms; negative time is before pinch off, positive time after pinch off. (a) Medium resolution simulation and (b) experiment from Longuet-Higgins et al. [22], reproduced with permission of M. Longuet-Higgins. 


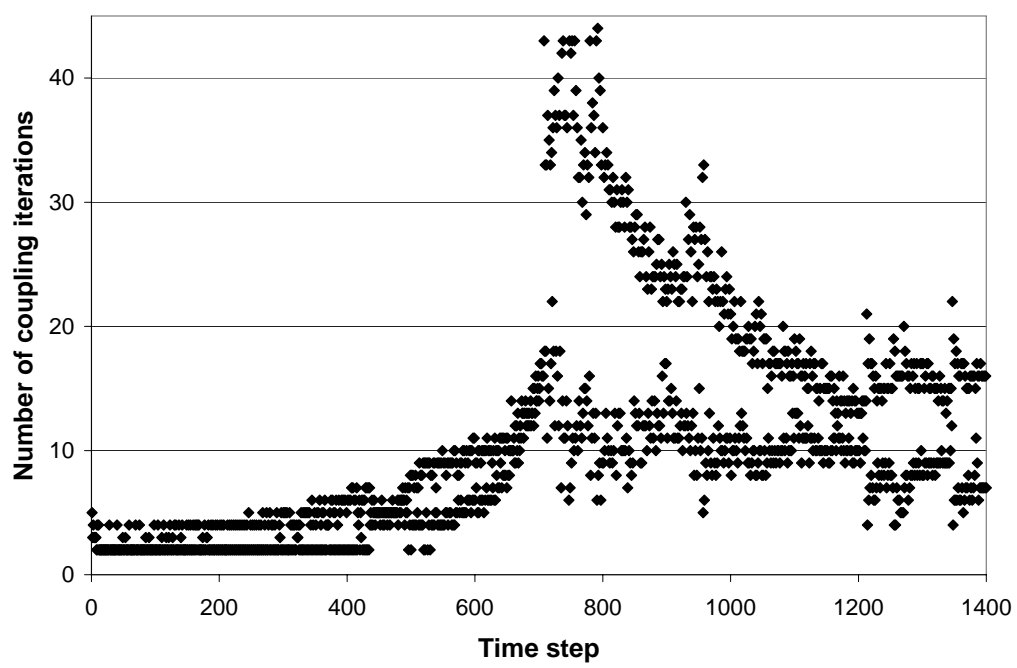

(a)

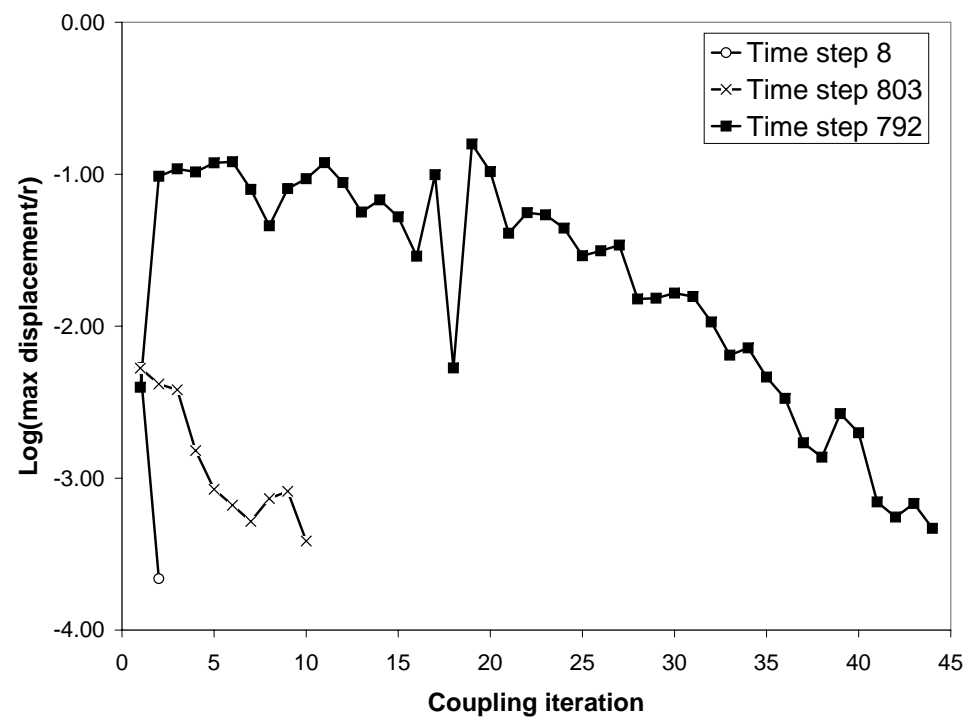

(b)

Fig. 11. (a) The number of coupling iterations required to bring the maximal node displacement below $1 / 10^{3.3}$ of the needle radius each time step during the simulation of a bubble that pinches of in time step 705, with initial number of nodes on the interface $N=63$ and initial time step $\Delta t=\Delta T / 2(\Delta T=0.005 \mathrm{~s})$. (b) The convergence behaviour of the coupling iterations for time step 8 (2 iterations), time step 803 (10 iterations) and 792 (44 iterations). 
Table 1

\begin{tabular}{|r|rl|}
\hline$\rho_{1}$ & 998.2 & $\mathrm{~kg} / \mathrm{m}^{3}$ \\
$\mu_{1}$ & 0.001003 & $\mathrm{Pas}$ \\
$\sigma$ & 0.070 & $\mathrm{~N} / \mathrm{m}$ \\
\hline
\end{tabular}

Parameters used for the simulation of an oscillating droplet. 


\section{Table 2}

\begin{tabular}{|r|r||c|c||c|c|}
\hline$\Delta t$ & $N$ & $\hat{\omega}_{2}$ & Error & $\tau_{2}$ & Error \\
\hline$\Delta T$ & 21 & 764.98 & 1.56 & 0.00910 & 94.71 \\
$\Delta T / 2$ & 21 & 757.33 & 0.55 & 0.01680 & 90.24 \\
$\Delta T / 2$ & 42 & 756.50 & 0.44 & 0.01847 & 89.27 \\
$\Delta T / 2$ & 61 & 756.49 & 0.44 & 0.01849 & 89.26 \\
$\Delta T / 2$ & 81 & 756.44 & 0.43 & 0.01860 & 89.20 \\
$\Delta T / 16$ & 21 & 754.01 & 0.11 & 0.07578 & 56.00 \\
$\Delta T / 32$ & 21 & 753.43 & 0.03 & 0.10558 & 38.69 \\
$\Delta T / 32$ & 42 & 749.36 & 0.51 & 0.11821 & 31.36 \\
$\Delta T / 32$ & 81 & 751.20 & 0.27 & 0.12971 & 24.68 \\
\hline
\end{tabular}

Influence of the number of nodes on the interface on the angular frequency $\hat{\omega}_{2}$ and the time constant of the damping $\tau_{2}$ compared with the influence of the time step $\Delta t$, with $\Delta T=0.00035 \mathrm{~s}$. The Error is the relative difference with the Richardson extrapolation in Table 3. 


\begin{tabular}{|c|c|c|c|c|}
\hline$\Delta t$ & $\begin{array}{c}\hat{\omega}_{2} \\
{[\mathrm{rad} / \mathrm{s}]}\end{array}$ & $\begin{array}{c}\text { Error } \\
{[\%]}\end{array}$ & $\begin{array}{l}\tau_{2} \\
{[\mathrm{~s}]}\end{array}$ & $\begin{array}{c}\text { Error } \\
{[\%]}\end{array}$ \\
\hline$\Delta T$ & 764.98 & 1.56 & 0.00910 & 94.71 \\
\hline$\Delta T / 2$ & 757.33 & 0.55 & 0.01680 & 90.24 \\
\hline$\Delta T / 4$ & 761.19 & 1.06 & 0.02967 & 82.77 \\
\hline$\Delta T / 8$ & 755.13 & 0.26 & 0.04929 & 71.38 \\
\hline$\Delta T / 16$ & 754.01 & 0.11 & 0.07578 & 56.00 \\
\hline$\Delta T / 32$ & 753.43 & 0.03 & 0.10558 & 38.69 \\
\hline$\Delta T / 64$ & 753.34 & 0.02 & 0.13135 & 23.73 \\
\hline$\Delta T / 128$ & 753.28 & 0.01 & 0.14716 & 14.55 \\
\hline Richardson extrapolation & 753.21 & & 0.17221 & \\
\hline Lamb & 749.56 & 0.48 & 0.19884 & 15.47 \\
\hline
\end{tabular}

Table 3

Time step dependence study of the angular frequency $\hat{\omega}_{2}$ and time constant of the damping $\tau_{2}$ during the second period of a water droplet's oscillation. The number of nodes on the interface is $N=21$ in all simulations and $\Delta T=0.00035 \mathrm{~s}$. The Error is the relative difference with the Richardson extrapolation. 


\section{Table 4}

\begin{tabular}{|r||c|c|c|}
\hline$\Delta t$ & Minimal & Average & Maximal \\
\hline$\Delta T$ & 3 & 4.8 & 10 \\
$\Delta T / 2$ & 4 & 5.0 & 13 \\
$\Delta T / 4$ & 3 & 5.0 & 13 \\
$\Delta T / 8$ & 3 & 5.5 & 15 \\
$\Delta T / 16$ & 3 & 6.5 & 19 \\
$\Delta T / 32$ & 2 & 8.1 & 23 \\
$\Delta T / 64$ & 2 & 9.8 & 28 \\
$\Delta T / 128$ & 2 & 11.4 & 36 \\
\hline
\end{tabular}

The minimal, average and maximal number of coupling iterations required to bring the maximal node displacement below $1 / 10^{8}$ of the droplet radius for the simulations of the time step dependence study. The number of nodes on the interface is $N=21$ in all simulations and $\Delta T=0.00035 \mathrm{~s}$. Data from the previous time step are used $(l=1)$. 


\section{Table 5}

\begin{tabular}{|r||c|c|c|}
\hline$\Delta t$ & Minimal & Average & Maximal \\
\hline$\Delta T$ & 5 & 7.2 & 10 \\
$\Delta T / 2$ & 6 & 8.4 & 14 \\
$\Delta T / 4$ & 7 & 9.4 & 47 \\
\hline
\end{tabular}

The minimal, average and maximal number of coupling iterations required to bring the maximal node displacement below $1 / 10^{8}$ of the droplet radius for the first three simulations of the time step dependence study. The number of nodes on the interface is $N=21$ in all simulations and $\Delta T=0.00035 \mathrm{~s}$. No data from the previous time steps are used $(l=0)$. 


\section{Table 6}

\begin{tabular}{|c||c|c|c|}
\hline$N$ & Minimal & Average & Maximal \\
\hline 21 & 4 & 5.0 & 13 \\
42 & 4 & 5.1 & 11 \\
61 & 4 & 5.1 & 12 \\
81 & 4 & 5.2 & 16 \\
\hline
\end{tabular}

The minimal, average and maximal number of coupling iterations required to bring the maximal node displacement below $1 / 10^{8}$ of the droplet radius for the second simulation of the time step dependence study with different number of nodes $N$ on the interface. $\Delta t$ is $\Delta T / 2$ in all simulations, with $\Delta T=0.00035 \mathrm{~s}$. Data from the previous time steps are used $(l=1)$. 
Table 7

\begin{tabular}{|r|rr|}
\hline$p_{\infty}$ & $10^{5}$ & $\mathrm{~Pa}$ \\
$p_{C}$ & 100073 & $\mathrm{~Pa}$ \\
$\sigma$ & 0.070 & $\mathrm{~N} / \mathrm{m}$ \\
$\mu_{1}$ & $1.81 \cdot 10^{-5}$ & $\mathrm{Pas}$ \\
$\mu_{2}$ & 0.001003 & $\mathrm{Pas}$ \\
$\rho_{2}$ & 998.2 & $\mathrm{~kg} / \mathrm{m}^{3}$ \\
$\mathrm{~g}$ & 9.81 & $\mathrm{~m} / \mathrm{s}^{2}$ \\
\hline
\end{tabular}

Parameters used for the simulation of bubble growth and detachment from a vertical needle. 


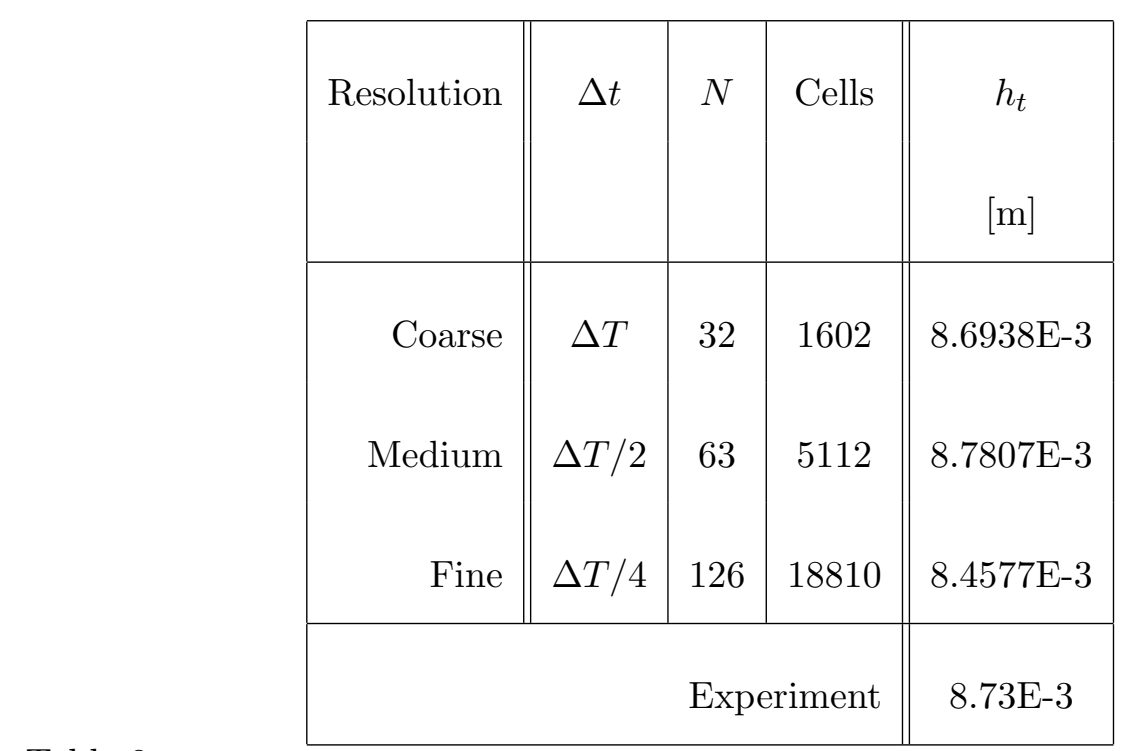

Table 8

Results from the time step and grid dependence study for the position of the bubble's top above the needle $h_{t}$ at detachment, with $\Delta T=0.005 \mathrm{~s}$. Experimental value from Longuet-Higgins et al. [22]. 